\title{
DFSS and Robust Optimization Tool for Multibody System with Random Variables*
}

\author{
Min-Soo KIM ${ }^{* *}$, HuiJe CHO ${ }^{* * *}$, Soon-Geul LEE ${ }^{* *}$, Jinhwan CHOI ${ }^{* *}$ \\ and DaeSung BAE ${ }^{* * * *}$ \\ ** Department of mechanical engineering, Kyunghee University, \\ Yongin, Gyeonggi-do, 449-791 South Korea \\ **inopl@functionbay.co.kr
}

*** FunctionBay, Inc., 6F, Industrial Bank Building, 246-5, Seohyeon-dong, Bundang-gu, Seongnam-si, Gyeonggi-do, 463-824 South Korea

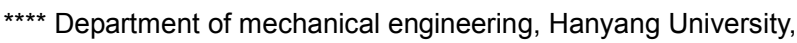
Ansan, Gyeonggi-do, 425-791 South Korea

\begin{abstract}
This study presents the DFSS optimization for the dynamic responses of a paper feeding mechanism. The flexible paper is idealized as a series of rigid bars connected by revolute joints and rotational spring dampers. In this mechanism, a paper is fed by a contact and friction mechanism on rollers or guides. The design objective is to minimize the slip amounts between paper and mechanisms and satisfy the 6-sigma constraint for the nip forces of rollers. In order to avoid the difficulty of design sensitivity analysis and overcome the numerical noise, a meta-model based optimization is employed. In this approach, first, the space filling methods and the classical DOE methods are used to generate sampling points. Second, the meta-models are constructed from the Kriging, RBF and RSM methods. Finally, a well-developed numerical optimizer sequentially solves the approximate optimization problem. In the numerical test, the DFSS for the paper feeding mechanism problem, having 8-random design variables, is solved in only 23 analyses.
\end{abstract}

Key words: DFSS, Optimization, Multibody, Meta-Model, Paper Feeding System

\section{Introduction}

Recently the media transport systems, such as printers, copiers, fax, ATMs, cameras, film develop machines, etc., have been widely used and being developed rapidly. In this field, it is an important key technology to determine kinematic mechanisms of parts dimensions, and materials, etc for the media machine developers. To shorten the develop time, reduce the cost, and improve the machine performance, most of early works has done to develop the computer simulation for analyzing the paper feeding and separation process. Among them, RecurDyn/MTT2D and RecurDyn/MTT3D are widely used in these areas.

Although the analysis process has well developed in the media transport systems, however, its' design optimization is very difficulty because the analytical design sensitivity process is very difficulty in the multi-body dynamics ${ }^{(1-3)}$. What is worse, a lower-pass filter are frequently used to signify the dynamic responses. In this case, analytical approach for design sensitivity is impossible.

This study introduces a meta-model based design optimization for dynamic response optimization, which can avoid the design sensitivity analysis and overcome the numerical noise. Especially, Design For Six Sigma (DFSS) and robust optimization is easily implemented by using the gradient information of meta-models. Section 2 reviews the early works for modeling the flexible paper and analyzing the media transport systems. Section 3 presents the proposed optimization strategy. Section 4 explains the DFSS optimization of a paper feeding mechanism. Finally, section 5 presents the conclusion of this study. 


\section{Review of paper modeling}

Since a thin plate model with an orthotropic material property suggested to model a paper behavior ${ }^{(4)}$, many numerical techniques have tried to analysis contact normal and frictional forces between paper and guide with respect to paper velocity and attack angle. Among them, beam elements are widely used to represent a flexible paper ${ }^{(5-6)}$. A separation mechanism of paper while developing numerical analysis models for a copy machine has been investigated ${ }^{(7-8)}$. The local static mechanics of electrometric nip system for media transport system has been introduced. The nonlinear finite element method and experimental measurement techniques are used to investigate the large deformable rollers. Several unique phenomena, such as skewing sheet, etc., of nip feeding system are well described $^{(9)}$. The stiffness of a coated paper has been computed ${ }^{(10)}$. The computer modeling techniques is introduced for the design and analysis of film feeding mechanisms. The primitive dynamic analysis of two-dimensional film-feeding models is presented by using commercial computer program ${ }^{(11)}$. Computational modeling techniques and a computer simulation tools for two- and three-dimensional film feeding mechanisms have been developed $^{(12-13)}$. The modeling techniques such as a mathematical representation of a flexible paper, guide and rollers and contact algorithm have been implemented on a commercial dynamics analysis program of RecurDyn/MTT2D and RecurDyn/MTT3D. An experimental way is investigated to estimate a slip between paper and roller in a simple paper feeding mechanism while validating the results of experiment and simulation. RecurDyn/MTT2D have been used to simulate ${ }^{(14)}$.

\section{Meta-model based optimization}

\subsection{Simultaneous Kriging Model}

Meta-Models such as RSM, Kriging and Radial Basis Function (RBF) are increasingly used to approximate expensive responses in engineering fields. RSM was introduced in the classical DOE, which used a polynomial type regression model. Hence, it required the rotatable characteristics for sampling points such as CCD and SCD. However, Kriging ${ }^{(15)}$ and radial basis function ${ }^{(16)}$ are Bayesian models. Hence, they used a space filled sampling points such as Latin hypercube or descriptive designs ${ }^{(17)}$.

Kriging models can be defined as a combination of a regression model plus a departure term:

$$
y=\mathbf{X} \boldsymbol{\beta}+z(\mathbf{x}),
$$

where $y$ is the approximate model, $\mathbf{X} \boldsymbol{\beta}$ is a polynomial type regression model, and $z(\mathbf{x})$ is a Gaussian random process with $N\left(0, \sigma^{2}\right)$. If the regression model (X⿳⺈) globally approximates the design space, the departure term $z(\mathbf{x})$ represents the localized deviations so that the Kriging model interpolates the $n_{s}$ sampled points. In my experience, the regression model plays an important role in design optimization especially for insufficient sampling points. The covariance matrix of $z(\mathbf{x})$ is given by

$$
\operatorname{Cov}\left[z\left(\mathbf{x}_{i}\right) z\left(\mathbf{x}_{j}\right)\right]=\sigma^{2} \mathbf{R}\left[R\left(\mathbf{x}_{i}, \mathbf{x}_{j}\right)\right],
$$

where $\mathbf{R}$ is the correlation matrix and $R\left(\mathbf{x}_{i}, \mathbf{x}_{j}\right)$ is the correlation function between any two of the $n_{s}$ sampled points. Hence, $\mathbf{R}$ is a $n_{s} \times n_{s}$ symmetric matrix with ones in the diagonal term. There are many correlation functions $R\left(\mathbf{x}_{i}, \mathbf{x}_{j}\right)$. Among them, the Gaussian type is widely used

$$
R\left(\mathbf{x}_{i}, \mathbf{x}_{j}\right)=\exp \left[\sum_{l=1}^{k} \theta_{l}\left|\mathbf{x}_{i}^{l}-\mathbf{x}_{j}^{l}\right|^{2}\right],
$$

where $\theta_{l}$ are the unknown correlation parameters to fit model. The estimates, $\tilde{y}(\mathbf{x})$ of the response $y(\mathbf{x})$ at the untried values of $\mathbf{x}$ are given by 


$$
\tilde{y}(\mathbf{x})=\mathbf{X}(\mathbf{x}) \tilde{\boldsymbol{\beta}}+\mathbf{r}^{T}(\mathbf{x}) \mathbf{R}^{-1}(\mathbf{y}-\mathbf{X}(\mathbf{x}) \tilde{\boldsymbol{\beta}}) .
$$

The correlation vector between $\mathbf{x}$ and the sampled points $\left\{\mathbf{x}_{1}, \mathbf{x}_{2}, \ldots, \mathbf{x}_{n_{s}}\right\}$ is given by:

$$
\mathbf{r}(\mathbf{x})^{T}=\left[R\left(\mathbf{x}, \mathbf{x}_{1}\right), R\left(\mathbf{x}, \mathbf{x}_{2}\right), \ldots, R\left(\mathbf{x}, \mathbf{x}_{n_{s}}\right)\right]^{T}
$$

In the estimates, the unknown coefficients of regression model is determined as

$$
\tilde{\boldsymbol{\beta}}=\left(\mathbf{X}^{T} \mathbf{R}^{-1} \mathbf{X}\right)^{-1}\left\{\mathbf{X}^{T} \mathbf{R}^{-1} \mathbf{y}\right\} .
$$

Also, in order to determine the unknown correlation parameters $\theta_{l}$, the estimate of the variance $\tilde{\sigma}^{2}$ (not the variance in the observed data) is introduced. Hence, the correction parameters $\theta_{l}$ is determined by solving

$$
\min _{\boldsymbol{\theta}>0}(\operatorname{det} \mathbf{R}(\boldsymbol{\theta}))^{1 / n_{s}} \tilde{\sigma}(\boldsymbol{\theta})
$$

While any values for $\boldsymbol{\theta}$ create an interpolation model, the best kriging model is found by solving the k-dimensional unconstrained optimization problems described in the above.

From the viewpoint of numerical optimization, equation (7) can be non-smooth because the correlation matrix $\mathbf{R}(\boldsymbol{\theta})$ is frequently singular during optimization process. Hence, some special techniques are required to avoid the singular phenomena and non-linearity of it. Hence, we use a singular value decomposition (SVD) and normalization and scaling techniques. Also, multi-objective formulation is introduced in equation (7) to solve the multiple kriging models simultaneously. This approach uses only one correlation matrix $\mathbf{R}(\boldsymbol{\theta})$ even for multiple kriging models ${ }^{(17)}$.

\subsection{DFSS and robust optimization formulation}

Lets' consider the general optimization formulation. Fundamentally, all the functions are composed of meta-models.

$$
\begin{array}{cl}
\text { Minimize } & \Psi(\mathbf{x})=\alpha \cdot f(\mathbf{x})+k_{0} \cdot \sigma_{0}(\mathbf{x}) \\
\text { subject to } & h_{i}(\mathbf{x})=0, i=1,2, \ldots, l \\
& g_{j}(\mathbf{x})+k_{j} \sigma_{j}(\mathbf{x}) \leq 0, j=1,2, \ldots, m \\
& \mathbf{x} \pm \sigma \in \Omega
\end{array}
$$

where $\sigma_{j}(\mathbf{x}), j=0,1, \ldots, m$ are the standard deviation of $f$ and $g$ that evaluated from meta-models. The value of $\alpha$ and $k_{i}$ are alpha weight and robust index, respectively. If $\alpha=0$ and $k_{0}=1$, the design objective is a minimization of the variance of $f(\mathbf{x})$. If $k_{j}=6$ is defined, the inequality constraints become DFSS constraints. If multiple objectives are given, the objective function of equation (8) is replaced by a preference function as

$$
\text { Minimize } P\left(\Psi_{k}(\mathbf{x})\right), \quad k=1,2, \ldots N_{o b j}
$$

In order to represent our preference function, let's consider following two objectives.

$$
\min _{\mathbf{x}} \Psi_{1}(\mathbf{x}) \text { and } \max _{\mathbf{x}} \Psi_{2}(\mathbf{x})
$$

There are many preference functions in multi-objective optimization strategy. Among them, we uses following two types. 


$$
\begin{aligned}
& P(\mathbf{x})=w_{1}\left(\frac{\Psi_{1}(\mathbf{x})}{\eta \Psi_{1}^{G}}-1\right)+w_{2}\left(1-\frac{\Psi_{2}(\mathbf{x})}{\nu \Psi_{2}^{G}}\right) \\
& P(\mathbf{x})=\max \left\{w_{1}\left(\frac{\Psi_{1}(\mathbf{x})}{\eta \Psi_{1}^{G}}-1\right), w_{2}\left(1-\frac{\Psi_{2}(\mathbf{x})}{\nu \Psi_{2}^{G}}\right)\right\}
\end{aligned}
$$

where, the values of $w_{i}$ are the user defined weighting coefficients and the relaxation factors $\eta$ and $v$ are automatically determined. Also, the ideal solution $f_{i}^{G}$ is internally determined.

\subsection{Numerical optimization process}

The approximate optimization problems, based on meta-modes, are sequentially solved with augmented Lagrange multiplier method ${ }^{(3)}$. In order to avoid the convergence difficulty for the insufficient sampling points, the initial design is selected from the best points in the given DOE and move limit is automatically adjusted. Also, the polynomial types are automatically switched to the degree of convergence of optimization process.

In the first iteration, the sequential approximate optimization (SAO) process requires the sampling points. We provide a discrete Latin hypercube design, incomplete small composite design- ${ }^{(1)}$, incomplete small composite design-II, generalized small composite design and other classical DOE methods such as CCD and BBD etc. ${ }^{(17)}$.

In the subsequent iteration of SAO, a new optimal design is given. Next, exact analysis is done for this point. Then, this new information is added to the design database. Hence, meta-model is newly developed and repeat these processes until the convergence criterion is satisfied for their tolerance values.

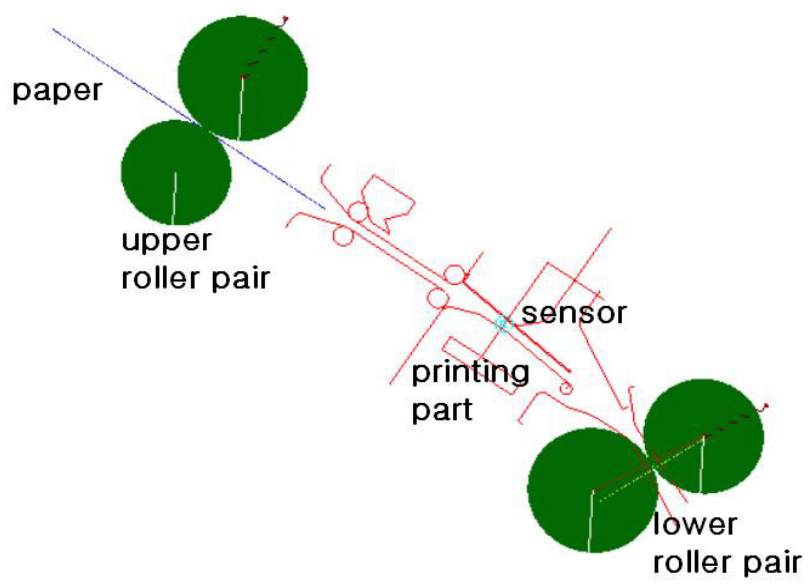

Figure 1. A paper feeding system model in Recurdyn/MTT2D

\section{DFSS of the paper feeding system}

\subsection{System Analysis}

Figure 1 shows a simple model of a paper feeding mechanism in the Recurdyn/MTT2D, which includes a printing part, feeding part and output part. In the mechanism, the upper part roller feeds the paper to the lower part roller. When the end part 
of feeding paper passes the sensor, the lower part roller rotates in the reverse direction. Then, the paper is feed to the upper part roller. During this reverse feeding process, the printing part is operated. The printing quality is fully depends on the slip of the feeding paper. Larger nip force can reduce the slip amount but manufacturing cost is increased. Hence, the slip amounts should be minimize within the allowable nip force limitation. The flexible paper is composed of rigid bars, revolute joints and rotational springs and dampers. Thus, the dynamic responses have numerical noise due to non-smoothness between bars. Hence, we use a lower-pass filter is needed to signify them.

\subsection{Random design variable selection}

Figure 2 shows the 8 random design variables. The $1^{\text {st }}$ through $3^{\text {rd }}$ design variables are Nip spring stiffness, damping coefficient and the initial pre-load in the lower part roller pair. The $4^{\text {th }}$ through $6^{\text {th }}$ design variables are the same characteristics in the upper roller pair. Also, in order to control the feeding direction in the lower part roller pair, the installation position and angles of moving roller are selected as the $7^{\text {th }}$ and $8^{\text {th }}$ design variables. The lower and upper limits on the design variables are listed in Table 1 side by side. All the design variables are regarded as random variables. The nip spring data has $\pm 5 \% \mathrm{COV}$, the roller position has $\pm 0.1(\mathrm{~mm})$ deviation and the roller angle has $\pm 0.1(\mathrm{deg}$.) deviation.

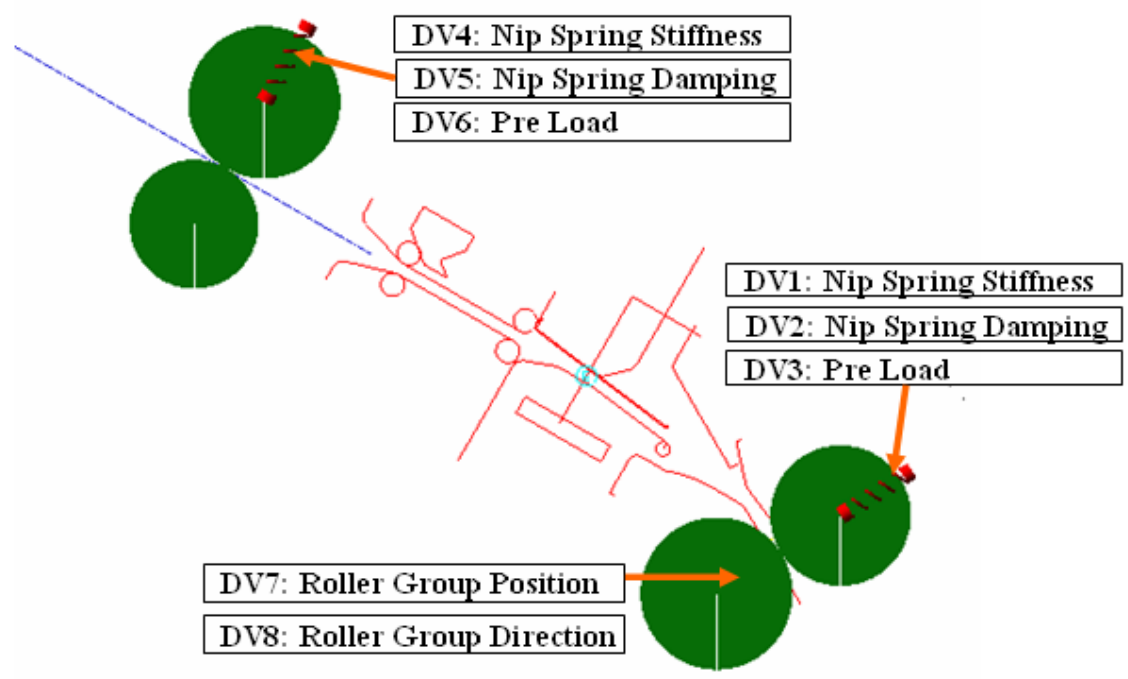

Figure 2 Random Design variables

\subsection{Design formulation}

Now, in order to enhance the printed quality of printer, the slip between the lower part roller pairs and the feeding paper should be minimized. Also, the nip force should be less than $0.025(\mathrm{~N})$ within 6 -sigma variance. Hence, the performance indexes are selected as the slip amounts and nip force during the reverse rotation of the lower part roller system.

Minimize the average of slips and the sum of slips

subject to $F_{n i p}^{\max }+6 \sigma\left(F_{n i p}^{\max }\right) \leq 0.025(N)$

in the design variable limits.

In the practical implementation, RecurDyn/AutoDesign ${ }^{(17)}$ uses a lower-pass filter to remove the numerical noise. 


\subsection{Optimization results}

From the viewpoint of meta-modeling constructions, the 8 design variable problem is quite large design. Hence, a discrete Latin-hypercube design is employed for the initial sampling in RecurDyn/AutoDesign. First, the 16 sampling points are selected in the design range given in Table 1 and the current design is added. Hence, total 17 sampling points is used. The performance index for the 17 sampling points are listed in Table 2. It is noted that the nip force values do not include 6-sigma values. Nevertheless, most of trials is greater than $0.025(\mathrm{~N})$.

Table 1. Lower and upper bounds on design variables

\begin{tabular}{lrr}
\hline & Lower bound & Upper bound \\
\hline DV1 & $6.00 \mathrm{E}-4$ & $1.50 \mathrm{E}-3$ \\
DV2 & $6.00 \mathrm{E}-5$ & $1.50 \mathrm{E}-4$ \\
DV3 & $2.00 \mathrm{E}-3$ & $8.00 \mathrm{E}-2$ \\
DV4 & $6.00 \mathrm{E}-4$ & $1.50 \mathrm{E}-3$ \\
DV5 & $6.00 \mathrm{E}-5$ & $1.50 \mathrm{E}-4$ \\
DV6 & $2.00 \mathrm{E}-3$ & $8.00 \mathrm{E}-2$ \\
DV7 & $-3.00 \mathrm{E}+0$ & $3.00 \mathrm{E}+0$ \\
DV8 & $-1.00 \mathrm{E}+0$ & $1.00 \mathrm{E}+0$ \\
\hline
\end{tabular}

Table 2 Performance index for the initial design

\begin{tabular}{cccc}
\hline Trials & Average of Slip & Sum of Slip & Nip Force \\
\hline 1 & $2.04 \mathrm{E}+00$ & $4.10 \mathrm{E}+02$ & $5.39 \mathrm{E}-02$ \\
2 & $7.26 \mathrm{E}-01$ & $1.45 \mathrm{E}+02$ & $7.33 \mathrm{E}-02$ \\
3 & $4.56 \mathrm{E}+00$ & $8.98 \mathrm{E}+02$ & $3.26 \mathrm{E}-02$ \\
4 & $2.80 \mathrm{E}+00$ & $5.60 \mathrm{E}+02$ & $3.75 \mathrm{E}-02$ \\
5 & $1.33 \mathrm{E}+00$ & $2.66 \mathrm{E}+02$ & $6.55 \mathrm{E}-02$ \\
6 & $1.20 \mathrm{E}+00$ & $2.42 \mathrm{E}+02$ & $1.68 \mathrm{E}-02$ \\
7 & $1.09 \mathrm{E}+00$ & $2.17 \mathrm{E}+02$ & $2.19 \mathrm{E}-02$ \\
8 & $3.60 \mathrm{E}+00$ & $7.23 \mathrm{E}+02$ & $2.69 \mathrm{E}-02$ \\
9 & $9.64 \mathrm{E}-01$ & $1.94 \mathrm{E}+02$ & $7.15 \mathrm{E}-02$ \\
10 & $1.72 \mathrm{E}+00$ & $3.45 \mathrm{E}+02$ & $5.74 \mathrm{E}-02$ \\
11 & $1.32 \mathrm{E}+00$ & $2.59 \mathrm{E}+02$ & $5.33 \mathrm{E}-02$ \\
12 & $1.17 \mathrm{E}+01$ & $2.16 \mathrm{E}+03$ & $1.19 \mathrm{E}-02$ \\
13 & $9.52 \mathrm{E}-01$ & $1.89 \mathrm{E}+02$ & $6.17 \mathrm{E}-02$ \\
14 & $5.04 \mathrm{E}+00$ & $9.73 \mathrm{E}+02$ & $6.47 \mathrm{E}-03$ \\
15 & $2.47 \mathrm{E}+00$ & $4.96 \mathrm{E}+02$ & $4.28 \mathrm{E}-02$ \\
16 & $5.98 \mathrm{E}-01$ & $1.20 \mathrm{E}+02$ & $6.84 \mathrm{E}-02$ \\
17 & $2.03 \mathrm{E}+00$ & $4.08 \mathrm{E}+02$ & $4.76 \mathrm{E}-02$ \\
\hline
\end{tabular}

In this study, simultaneous Kriging models combined with pure quadratic polynomials are employed for meta-models. RecurDyn/AutoDesign requires 6 iterations. The iteration summary is listed in Table 3. The final design successfully satisfies the nip force constraints and reduces the slip amounts.

Table 3 Performance index from SAO

\begin{tabular}{cccc}
\hline SAO & Average of Slip & Sum of Slip & Nip Force \\
\hline 1 & 4.727 & 945.4 & $2.135 \mathrm{E}-02$ \\
2 & 1.216 & 242.1 & $1.899 \mathrm{E}-02$ \\
3 & 1.166 & 234.4 & $2.070 \mathrm{E}-02$ \\
4 & 1.096 & 220.4 & $1.927 \mathrm{E}-02$ \\
5 & 1.091 & 218.2 & $2.034 \mathrm{E}-02$ \\
6 & 1.088 & 217.6 & $2.019 \mathrm{E}-02$ \\
\hline
\end{tabular}


The convergence criteria are selected as the relative change of objectives between consecutive iterations and the maximum violation of constraints. Their convergence tolerances are selected as 0.01, respectively. RedurDyn/AutoDesign provides the approximate value of standard deviation for the performance index. Then, the DFSS constraint violation is checked as

$$
\text { violation : } \mu+6 \tilde{\sigma} \leq \text { limit . }
$$

The approximate standard deviation for nip force is given as 0.0008362 . Thus, the violation values is evaluated as $0.02019-6 * 0.0008362$, which is less than the limit within its' convergence tolerance. Figure 3 shows the convergence history of SAO.

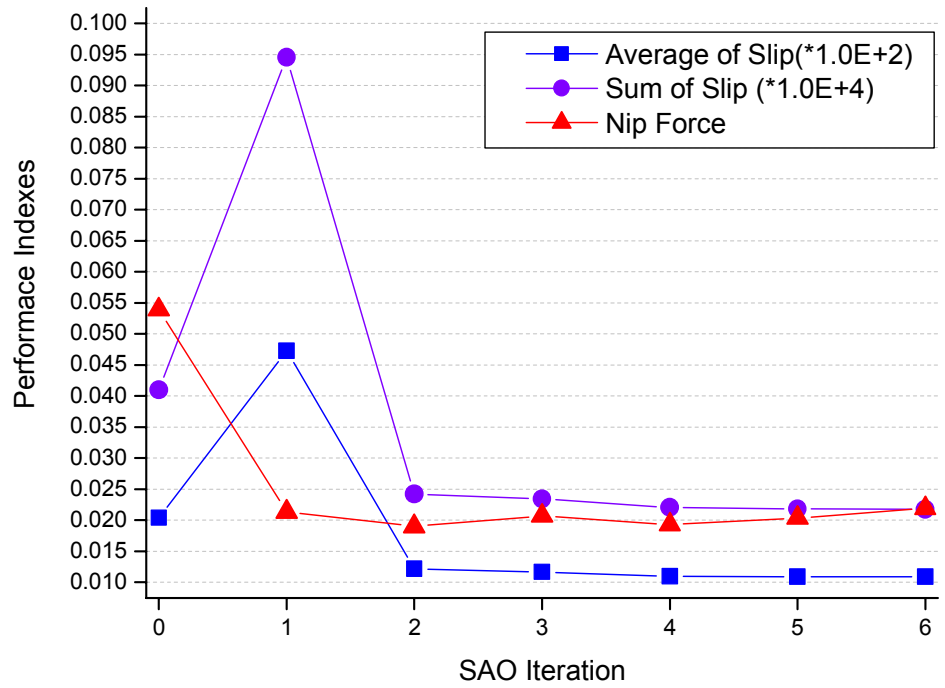

Figure 3 Convergence history of SAO

Also, in order to validate the DFSS results, we check the constraint violation by using the sampled variance. To do this, 10 points are sampled from a latin-hypercube method in the neighborhood of the final design. The sampled range is random variable deviation. The sampled standard deviation is evaluated from the final design and additional 10 values. The sample standard deviation is obtained as 0.00050226 . This is less than the approximate standard deviation. It represents that the proposed design satisfies the 6-sigma constraints by using only 23 evaluations.

For the initial and final designs, the nip forces are shown in Fig. 4 and 5. The final design is much less than the initial. These comparisons show the role of a lower-pass filter. Figure 6 shows the additional 10 analysis results for DFSS validation.

Finally, the final design values are $(8.00 \mathrm{E}-4,1.19 \mathrm{E}-4,1.59 \mathrm{E}-4,1.24 \mathrm{E}-3,6.00 \mathrm{E}-5$, $8.00 \mathrm{E}-2,1.0,2.165)$. Also, the initial design values are $(1.00 \mathrm{E}-3,1.00 \mathrm{E}-4,5.00 \mathrm{E}-2,1.00 \mathrm{E}-3$, $1.00 \mathrm{E}-4,5.00 \mathrm{E}-2,0.0,0.0)$. In this comparison, it is noted that the installation position and angles are changed. 


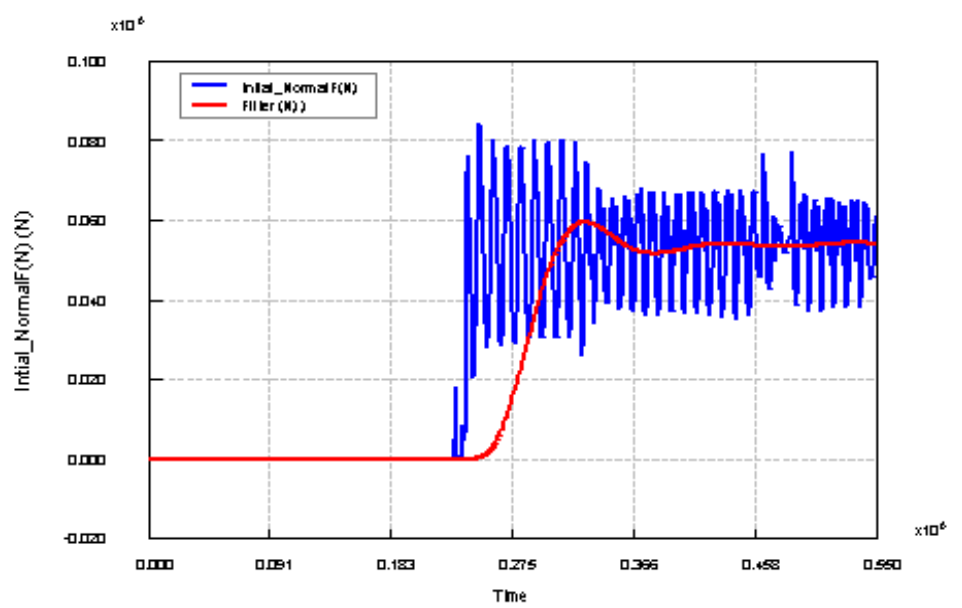

Figure 4 Nip force for the initial design

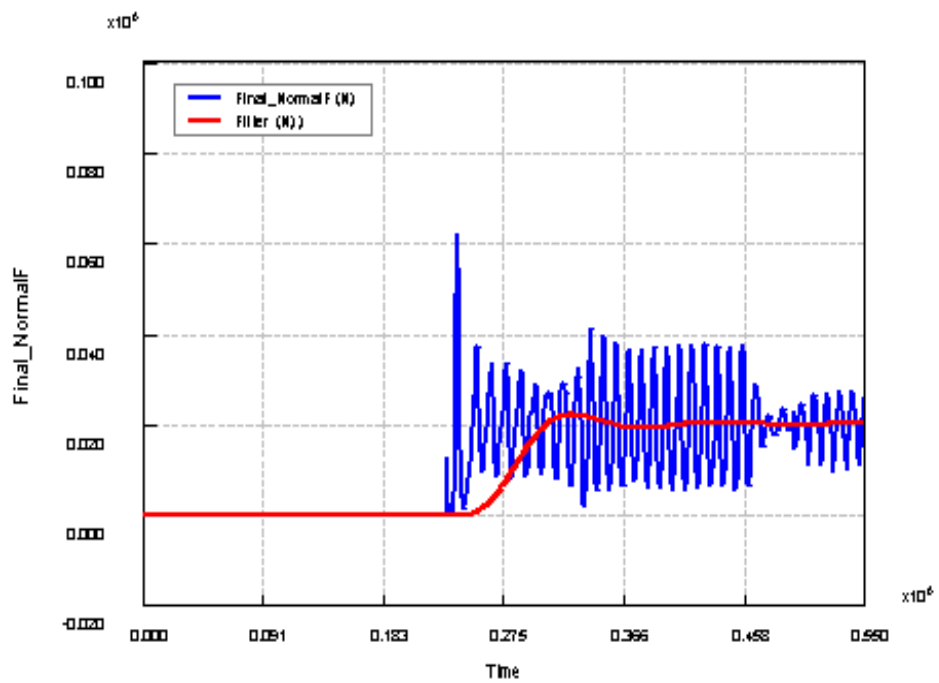

Figure 5 Nip force for the final design

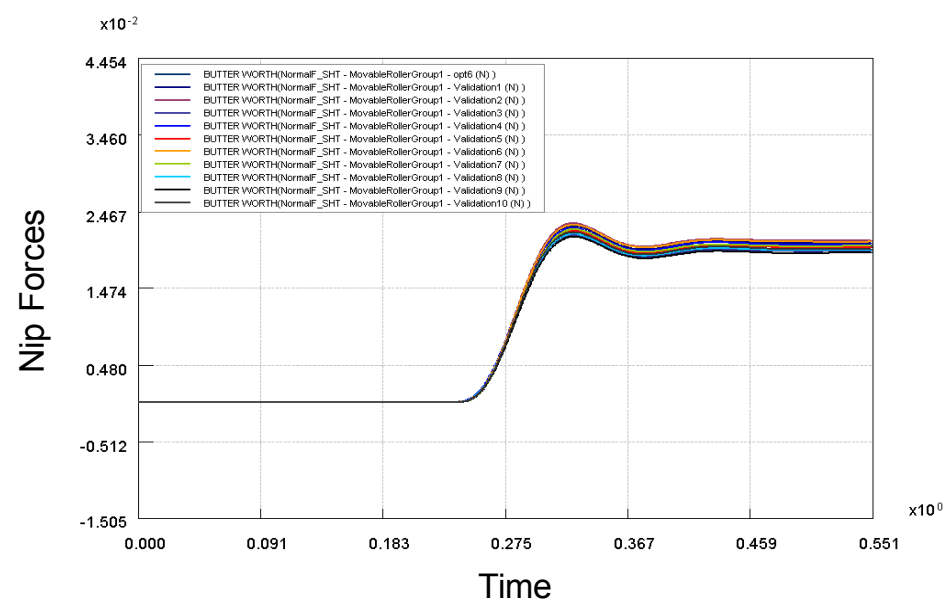

Figure 6 Nip force for the additional 10 analysis used in DFSS validation 


\section{Concluding remarks}

This study introduces the meta-model based design strategy for dynamic response optimization. This can avoid the difficulty of design sensitivity analysis, especially when a lower-pass filter is employed. Also, it shows that DFSS can be easily implemented by using the approximate variance from meta-model. In the numerical test, it successfully solved for 6-sigma design of the paper feeding mechanism only for 23 analyses including the initial samplings. Finally, the DFSS optimization results are validated by the sampled variance.

\section{Acknowledgement}

Some of researchers of this research got financial support of the second stage of BK21 program and the Basic Research Fund of the Agency for Defense and Development Grant No. ADD-04-05-02.

\section{References}

(1) Kim, M.-S. and Heo, S.-J., Conservative quadratic RSM combined with incomplete small composite design and conservative least squares fitting, KSME International Journal, Vol. 17, No. 5, pp. 698 702, 2003

(2) Kim, M-S and Choi, D.-H. An efficient dynamic response optimization using the design sensitivities approximated within the estimate confidence radius, KSME International Journal, Vol. 15, No. 8, pp. 1143 1155, 2001.

(3) Kim M.-S and Choi, D.-H., Min-max dynamic response optimization of mechanical systems using approximate augmented Lagrangian, Int. J. Numer. Meth. Engng., Vol 43, pp. 549 564, 1998.

(4) Thorpe, J.L., Paper as an orthotropic thin plate, Tappi, Vol. 64, No.3, pp. 119-121, 1981.

(5) Stolte, J. and Benson, R.C., Dynamic Deflection of Paper Emerging from a Channel, ASME Journal of Vibration and Acoustics, Vol. 114, pp. 187-193, 1992.

(6) Stolte, J. and Benson, R.C., And Extending Dynamic Elastica: Impact With a Surface, ASME Journal of Vibration and Acoustics, Vol. 115, pp. 308-313, 1993.

(7) Stack, K. D., A Study of Friction Feed Paper Separation, Transaction of the ASME-B-Journal of Engineering for Industry, Vol. 115, pp. 236-241, 1993.

(8) Stack, K. D, Stole, J. and Benson, R. C., A review of computer simulation models for sheet, Advances in information storage systems, Vol. 10, pp. 173-184, 1999.

(9) Diehl, T., Two dimensional and three dimensional analyses of nonlinear nip mechanics with hyperelastic material formulation, Ph. D. Thesis, University of Rochester, Rochester, NewYork, 1995.

(10) Koji, O., Toshiharu, E. and Fumihiko, O., Evaluation and Control of Coated paper Stiffness, Proceedings of Tappi advanced coating fundamentals symposium, Tappi press, Atlanta, USA, pp. 121-132, 1999.

(11) Ashida, T., The Meeting Material of The Japan Society for Precision Engineering, Japan, 2000.

(12) Cho, H. J., Bae, D. S., Choi, J. H. and Suzuki, T., Dynamic Analysis and Contact Modeling for Two Dimensional Media Transport System, Proceedings of DETC'03, DETC2003/MECH-48338, ASME 2003 Design Engineering Technical Conferences and Computers and Information in Engineering Conference, Chicago, Illinois, USA, September 2-6, 2003.

(13) Cho, H. J., Bae, D. S., Choi, J. H., Lee, S. G. and Rhim, S. S., Simulation and Experimental Methods for Media Transport System: Part I, Three-Dimensional Sheet Modeling Using Relative Coordinate, Journal of Mechanical Science and Technology, Vol. 19, No. 1, pp. 305 311, 2005.

(14) Ryu, J. K., Song, I. H., Lee, S. G., Rhim, S. S. and Choi, J. H., Simulation and Experimental Methods for Media Transport System: Part II, Effect of Normal Force on Slippage of Paper, Proceedings of ACMD'04, The Second Asian Conference on 
Multibody Dynamics 2004, Olympic Parktel, Seoul, Korea August 1-4, 2004.

(15) Farhang-Mehr, A. and Azarm, S., Bayesian meta-modeling of engineering design simulations: A sequential approach with adaptation to irregularities in the response behaviour, Int. J. Numer. Meth. Engng., Vol. 62, pp. 2104 2126, 2005.

(16) Wang, J.G. and Liu, G.R., A point interpolation meshless method based on radial basis functions, Int. J. Numer. Meth. Engng., Vol. 54, pp. 1623 1648, 2002

(17) Kim, M.-S., RecurDyn/AutoDesign: Meta-Model based design optimizer, Theoretical Manual, FunctionBay, 2006. 